\title{
Théorie de la définition et jeux vidéo : règles et lois dans l'expérience vidéoludique
}

Theory of Definition and Video Games: Rules and Laws in the Video Game

Experience

\section{Gauvain Leconte}

\section{(2) OpenEdition}

\section{Journals}

Édition électronique

URL : https://journals.openedition.org/sdj/1662

DOI : $10.4000 /$ sdj. 1662

ISSN : 2269-2657

Éditeur

Laboratoire EXPERICE - Centre de Recherche Interuniversitaire Expérience Ressources Culturelles

Education

\section{Référence électronique}

Gauvain Leconte, «Théorie de la défınition et jeux vidéo : règles et lois dans l'expérience vidéoludique », Sciences du jeu [En ligne], 11 | 2019, mis en ligne le 15 avril 2019, consulté le 12 décembre 2022. URL : http://journals.openedition.org/sdj/1662 ; DOI : https://doi.org/10.4000/sdj. 1662

Ce document a été généré automatiquement le 12 décembre 2022.

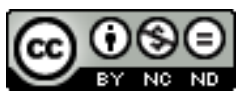

Creative Commons - Attribution - Pas d'Utilisation Commerciale - Pas de Modification 4.0 International - CC BY-NC-ND 4.0

https://creativecommons.org/licenses/by-nc-nd/4.0/ 


\section{Théorie de la définition et jeux vidéo : règles et lois dans l'expérience vidéoludique}

Theory of Definition and Video Games: Rules and Laws in the Video Game

Experience

Gauvain Leconte

1 La définition du concept de jeu a déjà une longue histoire derrière elle, depuis les définitions exposées dans les travaux pionniers de Huizinga (1988) et de Caillois (1967) jusqu'à celles proposées par Salen et Zimmerman (2004) ou Juul (2011 [2005]) - qui cherchent à condenser celles de leurs prédécesseurs - en passant par celles qui apparaissent dans le dialogue The Grasshopper de Suits (1978), dans l'ouvrage de game design de Crawford (1982) ou dans les recherches ethnologiques de Malaby (2005).

2 Mais on pourrait croire qu'une fois la question de la définition du concept de jeu réglée, celle du jeu vidéo irait de soi : comme la bande dessinée, le jeu vidéo semble porter sa définition dans son nom : c'est un jeu dont la spécificité serait de se dérouler sur un écran vidéo en permettant des interactions avec les joueuses ${ }^{1}$ via un dispositif algorithmique. Pourtant, le concept même de jeu vidéo a suscité lui aussi un débat au fur et à mesure que les jeux vidéo sont devenus des objets de recherche. Ces questions de définition ont en effet eu un rôle central dans l'émergence d'un champ d'études consacrées spécifiquement aux jeux vidéo (les gamestudies) puisqu'il semblait indispensable de circonscrire précisément l'ensemble des jeux vidéo pour élaborer une théorie de ces objets. Dans cette optique, les jeux ont souvent été définis comme des artefacts, c'est-à-dire des systèmes de règles permettant l'émergence de nombreuses possibilités d'action dont certaines sont valorisées parce qu'elles mènent à la victoire et les critiques de cette approche des games studies ont ainsi été amenées à reposer la question de la définition des jeux et des jeux vidéo ${ }^{2}$.

3 Mais il faut remarquer qu'à de rares exceptions près, ces débats définitionnels portent tous sur le contenu des définitions du jeu vidéo, et non sur leur forme, c'est-à-dire sur 
le type de définition qui serait adapté à l'objet «jeu vidéo ». Il semble implicitement admis par les chercheuses qui traitent des questions définitionnelles qu'une définition devrait capter l'essence de l'espèce jeu vidéo, c'est-à-dire trouver une propriété commune à tous les jeux vidéo ou énoncer des critères nécessaires et suffisants pour inclure un objet dans l'extension du concept de jeu vidéo.

Or, cette acception des définitions ne va pas de soi et a fait l'objet de nombreux travaux en philosophie et en logique depuis (au moins) Le Politique de Platon, où les interlocuteurs du dialogue cherchent à trouver une définition de l'être humain en utilisant la méthode dialectique. La philosophie et la logique peuvent ainsi aider à renouveler les questions définitionnelles portant sur les notions de jeu et de jeu vidéo en les abordant non seulement sous l'angle de leur contenu, mais aussi de leur forme ${ }^{3}$.

C'est pourquoi, avant d'aborder directement les problèmes liés à la définition de la notion de jeu vidéo dans la deuxième section de cet article, je consacre la première section à un passage en revue de différents types de définitions utiles pour traiter ces problèmes. Dans la troisième section, je propose de définir le jeu vidéo comme un concept à texture ouverte. La quatrième section montre comment ce type de définition permet de traiter les problèmes exposés dans la deuxième section. Elle examine aussi les rapprochements que cette définition permet de faire entre le jeu vidéo et d'autres activités ludiques, comme le sport. La cinquième et dernière section prolonge ces analyses en montrant que l'expérience vidéoludique consiste en partie à découvrir des mondes possibles structurés par des lois différentes de celles que l'on expérimente quotidiennement.

\section{La question de la forme des définitions}

\section{Définitions explicites et définitions implicites}

6 Même si les questions de définitions occupent les philosophes, les logiciens et les mathématiciens depuis l'Antiquité, l'origine de la théorie moderne des définitions se trouve dans la controverse qui a opposé Frege et Hilbert au sujet de l'axiomatique de la géométrie que celui-ci avait proposée en 1899 dans ses Fondements de la géométrie. Parmi les différentes critiques adressées à l'ouvrage d'Hilbert, Frege remarque qu'« au sein des propositions mathématiques, [il] aimerai[t] séparer les définitions de tout le reste (axiomes, lois fondamentales, théorème)» car «les propositions non définitionnelles ne doivent contenir aucun mot ou signe dont le sens et la référence ne soit pas déjà pleinement établi » (in Rivenc, 1992, p. 222).

7 En réponse, Hilbert soutient que, par exemple, les axiomes du prédicat "entre» constituent la définition de ce prédicat, parce qu'ils donnent les caractéristiques (Merkmale) que réclame Frege :

ces caractéristiques sont données en détail dans les axiomes II1-II5. Néanmoins si l'on tient à prendre le mot «définition » précisément dans le sens reçu il suffit de dire: "entre" est une relation entre points d'une droite qui possède les caractéristiques suivantes : II1...II5. » (Rivenc, 1992, p. 226).

Cette opposition entre Frege et Hilbert ouvre la question de savoir si la définition d'un terme doit être explicite ou peut rester implicitement contenue dans les axiomes de la 
théorie. La définition d'un prédicat n-aire $\mathrm{T}$ est dite « explicite » si et seulement si elle est une formule close de la forme suivante :

•(D) $\left[\mathrm{T}\left(\mathrm{x}_{1} \ldots \mathrm{x}_{\mathrm{n}}\right) \leftrightarrow \mathrm{U}\left(\mathrm{x}_{1} \ldots \mathrm{x}_{\mathrm{n}}\right)\right]$

$\mathrm{T}\left(\mathrm{x}_{1} \ldots \mathrm{x}_{\mathrm{n}}\right)$ est habituellement appelé le definiendum (terme défini), $\mathrm{U}\left(\mathrm{x}_{1} \ldots \mathrm{x}_{\mathrm{n}}\right)$ le definiens (terme qui définit). Une définition explicite est donc un énoncé présentant le signe d'équivalence « $\leftrightarrow$ » entre le definiens et le definiendum qui se remarque en langue naturelle par l'emploi de la formule «si et seulement si ». (D) se lit ainsi : «les $\mathrm{x}_{1} \ldots \mathrm{x}_{\mathrm{n}}$ sont (dans une relation) $\mathrm{T}$ si et seulement si les $\mathrm{x}_{1} \ldots \mathrm{x}_{\mathrm{n}}$ sont (dans une relation) $\mathrm{U} »$.

Pour que cette définition soit explicite, il faut que $U$ satisfasse les conditions suivantes :

- (c1) U est non-contradictoire

- (C2) U ne contient que les variables libres $x_{1} \ldots x_{n}$ présentes dans $T$.

- (C3) U ne peut faire intervenir que des termes primitifs ou déjà définis (T n'y apparaît pas).

Les conditions (C1-3) assurent que la définition présente un certain nombre de propriétés intéressantes. D'une part, si une définition est biconditionnelle et que le definiens contient les mêmes variables libres que le definiendum, alors(D) est la conjonction des deux énoncés conditionnels :

•(D') $\left[\mathrm{T}\left(\mathrm{x}_{1} \ldots \mathrm{x}_{\mathrm{n}}\right) \rightarrow \mathrm{U}\left(\mathrm{x}_{1} \ldots \mathrm{x}_{\mathrm{n}}\right)\right]\left(\right.$ si les $\mathrm{x}_{1} \ldots \mathrm{x}_{\mathrm{n}}$ sont $\mathrm{T}$ alors les $\mathrm{x}_{1} \ldots \mathrm{x}_{\mathrm{n}}$ sont $\left.\mathrm{U} . »\right)$

•(D") $\left[\mathrm{U}\left(\mathrm{x}_{1} \ldots \mathrm{x}_{\mathrm{n}}\right) \rightarrow \mathrm{T}\left(\mathrm{x}_{1} \ldots \mathrm{x}_{\mathrm{n}}\right)\right]\left(\right.$ ( si les $\mathrm{x}_{1} \ldots \mathrm{x}_{\mathrm{n}}$ sont $\mathrm{U}$ alors les $\mathrm{x}_{1} \ldots \mathrm{x}_{\mathrm{n}}$ sont $\mathrm{T}$. »)

(D') énonce une condition seulement nécessaire pour appliquer le terme $\mathrm{T}$ à des individus (tous les $x$ qui sont $\mathrm{T}$ sont aussi $\mathrm{U}$, mais il peut $\mathrm{y}$ avoir des $x$ qui sont $\mathrm{U}$ sans être T), (D") énonce une condition seulement suffisante pour appliquer le terme $\mathrm{T}$ à des individus (tous les $x$ qui sont $\mathrm{U}$ sont $\mathrm{T}$, mais il peut $\mathrm{y}$ avoir des $x$ qui sont $\mathrm{T}$ sans être $\mathrm{U}$ ). Autrement dit, une définition explicite fournit les conditions nécessaires et suffisantes à l'application du terme qu'elle définit et circonscrit précisément et rigoureusement l'extension de ce terme: c'est ce type de définition que recherchent la plupart des auteurs en quête d'une définition isolant l'essence du jeu ou du jeu vidéo.

Une autre propriété intéressante des définitions explicites est qu'elles permettent, comme le recommandait Pascal, de « substituer mentalement la définition à la place du défini » (Pascal, 1985, 70). En effet si le definiens n'est ni contradictoire ni circulaire, alors les définitions explicites respectent les critères de Lesniewski de non-créativité et d'éliminitavité (Suppes, 1957, 153) : le definiendum ne fait qu'abréger le definiens, il ne permet pas de prouver plus ou moins de choses que celui-ci.

On comprend alors que l'on cherche, quand cela est possible, des définitions explicites plutôt qu'implicites : elles ont le mérite de la rigueur et de la précision et peuvent être utilisées en toute sécurité. Plusieurs résultats de Padoa (1901) et de Beth (1962) montrent que l'on peut, sous certaines conditions ${ }^{4}$, transformer une définition implicite (à partir d'axiomes) en définition explicite. Il serait donc légitime et fécond de rechercher des définitions explicites de termes implicitement définis dans des théories, comme celui de « jeu » ou de « jeu vidéo ».

Mais les conditions en question sont très restrictives : le résultat de Beth ne vaut que pour des langages logiques du premier ordre (c'est-à-dire dans lesquels on ne peut quantifier que sur des variables et non sur des prédicats ou des relations), mais pas pour des langages d'ordres supérieurs et encore moins pour des langages naturels comme ceux que nous utilisons au quotidien, notamment pour parler de «jeu» ou de « jeu vidéo ». 


\section{Airs de famille et concepts à texture ouverte}

pourquoi la recherche d'autres formes de définitions que est pertinente lorsque l'on recherche la définition de termes employés dans le langage naturel. L'un des premiers à avoir reconnu cette nécessité est Wittgenstein qui, dans ses Investigations Philosophiques, rencontre des difficultés pour définir un des termes qui nous intéresse ici, celui de jeu :

66 - Considérons par exemple les processus que nous nommons les «jeux». J'entends les jeux de dames et d'échecs, de cartes, de balle, les compétions sportives. Qu'est-ce qui leur est commun à tous? - Ne dites pas : il faut que quelque chose leur soit commun, autrement ils ne se nommeraient pas « jeux » - mais voyez d'abord si quelque chose leur est commun. - Car si vous le considérez, vous ne verrez sans doute pas ce qui leur serait commun à tous, mais vous verrez des analogies, des affinités, et vous en verrez toute une série. [...] - Tous ces jeux sontils « divertissants »? Comparez les échecs et la marelle. Ou bien y a-t-il en tous une façon de gagner et de perdre, ou une compétition des joueurs? Songez aux jeux de patience. Dans les jeux de balle, on gagne et on perd ; mais quand un enfant lance la balle contre le mur et la rattrape, ce caractère se perd. [...] Et ainsi nous pouvons parcourir beaucoup d'autres groupes de jeux; voir surgir et disparaître des analogies.

Et tel sera le résultat de cette considération: nous voyons un réseau complexe d'analogies qui s'entrecroisent et s'enveloppent les unes les autres. (Wittgenstein, 1961, pp. 147-148)

Il faut souligner l'insistance de Wittgenstein dans ce passage sur le fait de s'efforcer de "voir» s'il y a quelque chose de commun entre les différents jeux au lieu de le présupposer : quand il s'agit de trouver une définition d'un terme du langage naturel, on ne peut pas partir de l'hypothèse qu'il existe une caractéristique qui serait propre à toutes les instances auxquelles s'applique ce terme - caractéristique dite " essentielle ", à la fois nécessaire et suffisante, que l'on retrouve dans les définitions explicites. Pour de tels termes, il est nécessaire de passer par une enquête empirique afin de déterminer si une telle caractéristique existe. Et l'exemple du mot « jeu » fournit à Wittgenstein un cas pour montrer que ce n'est pas une telle caractéristique unique, mais un réseau d'analogies qui lie les différentes formes de jeu entre elles, réseau d'analogie qu'il nomme « air de famille».

La position de Wittgenstein est parfois décrite comme " anti-essentialiste " (Tavinor, 2008), mais il ne faut pas la confondre avec une position hostile à toute définition (à moins de considérer, comme Carnap dans Testabilité et signification, que seules les définitions explicites méritent le nom de « définition »). Wittgenstein lui-même récuse cette interprétation dans les lignes qui suivent sa définition :

Comment le concept de jeu est-il délimité ? Qu'est-ce qui est encore jeu, qu'est-ce qui ne l'est plus? Pouvez-vous en indiquer les limites? Non. Vous pouvez en tracer quelques-unes : car aucune n'a encore été tracée. (Mais ceci ne vous a jamais gêné dans l'application du mot «jeu».) « Mais alors l'application de ce mot n'a pas de règle. » - Il n'est pas toujours délimité par des règles ; mais il n'y a pas non plus de règle au tennis qui prescrive jusqu'à quelle hauteur il est permis de lancer la balle, ni avec combien de force; et pourtant le tennis est un jeu qui a, lui aussi, ses règles. (Wittgenstein, 1961, pp. 148-149)

Autrement dit, ce n'est pas parce que l'on ne dispose pas de conditions nécessaires et suffisantes pour définir un terme que l'on ne peut pas en spécifier certaines conditions 
d'applications suffisantes. Il est par exemple bien connu que différents cas d'une même maladie peuvent présenter des symptômes très différents sans qu'aucun ne soit commun à tous les cas: il n'y a qu'un air de famille entre les symptômes de cette maladie. Cela n'empêche pas les médecins de définir les maladies à partir de leurs symptômes en utilisant des définitions polycritérielles, c'est-à-dire en les définissant comme des disjonctions de critères suffisants pour appliquer un terme médical à un cas particulier. C'est le cas notamment des définitions que l'on trouve dans le Diagnostic and Statistical Manual of Mental Disorders, le manuel publié par l'Association américaine de psychiatrie, qui dresse une liste de symptômes dont aucun n'est nécessaire ni suffisant pour identifier un trouble mental donné, mais qui spécifie combien de ces symptômes doivent être rassemblés pour que l'on puisse établir un diagnostic ${ }^{5}$.

Les «airs de famille » peuvent donc être définis par des définitions polycritérielles, c'est-à-dire par des disjonctions de critères suffisants pour appliquer un terme :

-(DP) $\left[\mathrm{U}\left(\mathrm{x}_{1} \ldots \mathrm{x}_{\mathrm{n}}\right) \vee \mathrm{V}\left(\mathrm{x}_{1} \ldots \mathrm{x}_{\mathrm{n}}\right) \vee \ldots \rightarrow \mathrm{T}\left(\mathrm{x}_{1} \ldots \mathrm{x}_{\mathrm{n}}\right)\right]\left(\right.$ si les $\mathrm{x}_{1} \ldots \mathrm{x}_{\mathrm{n}}$ sont $\mathrm{U}$ ou si les $\mathrm{x}_{1} \ldots \mathrm{x}_{\mathrm{n}}$ sont $\mathrm{V}$, ... alors les $\mathrm{x}_{1} \ldots \mathrm{x}_{\mathrm{n}}$ sont $\left.\mathrm{T} . »\right)$

21 Où « ...» indique la possibilité de rechercher et de trouver de nouvelles conditions suffisantes pour appliquer le terme T. Notons qu'il n'est pas requis que l'extension des prédicats $\mathrm{U}$ et $\mathrm{V}$ soient exclusives : elles peuvent se recouper et ainsi représenter « ce réseau complexe d'analogies qui s'entrecroisent et s'enveloppent les unes les autres » qui lie les différents exemples de jeux selon Wittgenstein.

Les arguments de Wittgenstein suggèrent que les définitions explicites ne sont pas forcément les plus adaptées si l'objectif que l'on se donne est de définir un terme déjà en usage dans un langage naturel pour désigner une réalité empirique car on risque de délimiter un domaine d'objet qui ne l'est pas et qui peut toujours s'étendre au fur et à mesure que de nouveaux objets sont découverts ou inventés. C'est précisément ce que remarque Waismann, un autre critique de la caractérisation traditionnelle des définitions, dans l'article où il introduit la notion de « concept à texture ouverte »:

Quoiqu'on fasse, aucun concept [empirique] n'est limité d'une manière telle qu'il n'y ait plus de place pour aucun doute. Lorsque nous introduisons un concept nous le limitons dans certaines directions. [...] Nous avons tendance à ne pas voir le fait que qu'il y a toujours d'autres directions dans lesquelles le concept n'a pas été défini.

(Waismann, 2004, p. 329)

Mais utiliser un concept à texture ouverte ne revient pas à renoncer à toute définition ni à considérer que seul un réseau de ressemblance peut expliquer l'usage d'un terme. L'objectif de Waissmann n'est pas de montrer qu'aucune définition des concepts empiriques (et particulièrement les concepts scientifiques) n'est possible, mais que ces concepts ne peuvent pas tous être réduits à des ensembles circonscrits de données d'observations qui suffiraient à vérifier son application :

La texture ouverte est une caractéristique tout à fait fondamentale de la plupart des concepts empiriques (mais non de tous), et c'est cette texture qui nous empêche de vérifier de façon concluante la plupart de nos énoncés empiriques. Prenez n'importe quel énoncé d'objet matériel. Les termes qui y figurent sont nonexhaustifs, ce qui signifie que nous ne pouvons pas prévoir complètement toutes les conditions possibles dans lesquelles ils seront utilisés. [...] Et cela veut dire que nous ne saurions prévoir complètement toutes les circonstances possibles dans lesquelles l'énoncé est vrai, ni celles dans lesquelles il est faux. (Waismann, 2004, p. 330)

Puisqu'en fait nous ne pouvons jamais éliminer la possibilité qu'émerge un facteur imprévu, nous ne pouvons jamais être tout à fait sûrs d'avoir inclus dans notre 
définition tout ce qui devrait l'être ; et, par conséquent, le processus de définition

d'affinage d'une idée continuera sans jamais atteindre son point final. (p. 332)

Comme on le voit, la forme syntaxique de (TO) diffère de celle de (DP) - les définitions polycritérielles - et de (D) - les définitions explicites. On a ainsi à notre disposition (au moins) trois formes de définitions différentes dont certaines - les définitions polycritérielles d'air de famille et les définitions de concept à texture ouverte semblent plus adaptées pour définir les concepts issus du langage naturel ou des enquêtes empiriques. Dans la section suivante, j'examine si ces apports de la théorie de la définition peuvent éclaircir les controverses sur la question de la définition du jeu vidéo.

\section{Problèmes de définitions}

\section{Problèmes des définitions explicites}

Si l'on envisage les différentes définitions du jeu et du jeu vidéo proposées, on s'aperçoit que, pour la plupart, elles se présentent sous la forme de définitions explicites. Salen et Zimmerman, dans leur ouvrage de référence sur le design de jeu (game design) consacrent un chapitre à détailler les différentes définitions du jeu qui ont été proposées par Parlett, Abt, Huizinga, Caillois, Suits, Crawford, Costikyan, Avedon et Sutton-Smith (Salen et Zimmerman, 2004, pp. 73-83). La définition de Salen et Zimmerman est fondée sur une comparaison de ces différentes définitions qui vise à exclure les caractéristiques qui leur semblent ne pas appartenir à tous les jeux ou appartenir à d'autres activités que le jeu. Leur « intention n'est pas de comprendre le fait de jouer (play) en tant que phénomène général, mais de démarquer clairement le domaine des jeux (games) et de la conception des jeux (game design)»(Salen et Zimmerman, 2004, p. 82). Leur définition prend alors la forme d'une définition explicite qui circonscrit un ensemble précis d'objets : « un jeu (game) est un système par lequel les joueuses s'engagent dans un conflit artificiel défini par des règles qui aboutit à un résultat quantifiable» (Salen et Zimmerman, 2004, p. 82, notre traduction). 
Juul procède de manière identique lorsqu'il examine, dans le chapitre 2 de Half-Real, les définitions de Huizinga, Caillois, Suits, Avedon et Sutton-Smith, Crawford, Kelley ainsi que Salen et Zimmerman pour construire son «modèle classique du jeu » autour de six caractéristiques (Juul, 2011 [2005], pp. 35-39) :

1. Un jeu (game) est un système formel fondé sur des règles,

2. ayant des variables et des résultats quantifiables,

3. où l'on assigne différentes valeurs aux différents résultats,

4. où la joueuse exerce un effort pour influencer les résultats,

5. où la joueuse est émotionnellement impactée par ces résultats,

6. et où les conséquences de cette activité sont optionnelles et négociables.

Pour Juul, « ces six caractéristiques du modèle sont nécessaires et suffisantes pour que quelque chose soit un jeu, c'est-à-dire que tous les jeux ont ces six caractéristiques et que posséder ces six caractéristiques est suffisant pour être un jeu » (Juul, 2011 [2005], p. 13). Il conçoit donc lui aussi sa définition du jeu comme une définition explicite.

30 Cependant, les approches de Juul et de Salen et Zimmerman diffèrent sur la question de la définition du jeu vidéo à partir de leur définition du jeu. Salen et Zimmerman considèrent que les jeux vidéo ont des caractéristiques qui sont présentes dans les autres jeux, mais que les jeux vidéo « incorporent de manière plus robuste » (Salen et Zimmerman, 2004, p. 90) : une interactivité immédiate, mais limitée, la manipulation d'information, des systèmes automatisés complexes et des communications en réseau. Cette définition du jeu vidéo reste intentionnellement vague : l'objectif de Salen et Zimmerman est moins de répondre à la question « que sont les jeux vidéo ? » qu'à celle «que peuvent les jeux vidéo?» Néanmoins il est clair que pour eux les jeux vidéo sont une espèce incluse dans le genre jeu: leur définition est apparentée aux définitions essentialistes aristotéliciennes par genre et différence spécifique.

D'autres définitions du jeu vidéo, même si elles n'ont pas le même contenu que celle de Salen et Zimmerman, adoptent la même forme. Par exemple, Nicolas Esposito propose la définition suivante : " Un jeu vidéo est un jeu auquel on joue à l'aide d'un dispositif audiovisuel et qui peut être fondé sur une histoire » (Esposito, 2005, notre traduction). Le jeu vidéo est l'espèce du genre jeu qui a pour différence spécifique d'impliquer un dispositif audiovisuel et d'ouvrir la possibilité à une narration.

Pour Juul, au contraire, les jeux vidéo ne forment pas qu'un sous-ensemble de la classe des jeux définie par son modèle : «l'histoire des jeux vidéo consiste en partie à rompre avec le modèle classique du jeu » (Juul, 2011 [2005], p. 14, notre traduction). En effet, les règles des jeux vidéo peuvent être plus complexes que celles des jeux, les résultats peuvent être plus variés, les actions des joueuses ne sont pas toujours valorisées, leurs efforts sont redirigés et les conséquences ne sont pas limitées de la même manière dans le temps et l'espace (Juul, 2011, p. 47). Wolf remarque lui aussi que sa définition des jeux vidéo comme « grille de pixels reconfigurable » élargit la catégorie des jeux à des programmes éducatifs ou de diagnostics (Wolf, p. 4, notre traduction). Karhulahti semble procéder de la même manière lorsqu'il propose la définition des jeux vidéo comme des «artefacts qui évaluent la performance» (Karhulahti, 2015, notre traduction), par opposition aux jeux non vidéo dans lesquels ce sont des individus (des arbitres notamment) qui évaluent la performance des joueuses. Pour ces auteurs, les jeux vidéo ne formeraient pas un sous-ensemble de la classe des jeux, mais une extension de la classe des jeux à d'autres objets ${ }^{6}$. 

jeux en général ou aux jeux non vidéo, les propositions de définition mentionnées cidessus ont pour point commun de rencontrer (au moins) trois difficultés, des cas limites qui entrent mal dans leurs définitions :

La première difficulté mérite le nom de "problème de Sim City ", tant cet exemple revient souvent la plume des théoriciens du jeu vidéo (Salen et Zimmerman, 2004, p. 82 ; Esposito 2005 ; Wolf, 2008, p. 4 ; Juul, 2011 [2005], p. 42 ; Karhulahti, 2015). Sim City (Maxis, 1989) est un jeu de gestion de ville dans lequel aucun objectif prédéfini n'est donné aux joueuses: il n'y a ainsi ni but à atteindre ni victoire définitive l'appellation de jeu "bac à sable ", pour traduire l'anglais sandbox, est souvent utilisée pour désigner les jeux vidéo qui partagent cette caractéristique, l'exemple récent le plus connu étant Minecraft (Mojang, 2011). Un tel jeu semble échapper à toute définition du jeu vidéo imposant comme condition nécessaire et suffisante les critères de performance, de compétition, de progression ou d'activité dirigée par un but. Les tenants de telles définitions rejettent donc ce jeu et tout ceux du même genre à la marge en les considérant comme des "jouets vidéo " (Karhulahti, 2015) plutôt que comme d'authentiques jeux.

2. On pourrait appeler la deuxième difficulté "problème de la transposition " parce qu'il concerne le cas de l'adaptation de jeux non vidéo en jeux vidéo comme les échecs, le Sudoku ou le jeu de plateau Risk (Tavinor, 2008 ; Juul, 2011 [2005], pp. 43-45). Tant que les règles sont les mêmes, il ne semble pas y avoir une grande différence entre jouer aux échecs sur un échiquier, par courrier ou sur un ordinateur, ni entre compléter un Sudoku dans un magazine ou sur un téléphone portable, ni entre faire une partie de Risksur le plateau de jeu ou sur Nintendo Switch (Ubisoft, 2018). Pourtant, les définitions ci-dessus qui traitent les jeux vidéo comme une espèce du genre jeu ou comme une classe à part de jeux distinguent clairement deux objets : les échecs sur échiquier et les échecs sur ordinateur, le Sudoku papier et le Sudoku numérique, le Risk sur plateau et le Risk sur console.

3. On peut rajouter un troisième cas problématique qui concerne l'adaptation de jeux non vidéo qui ont des règles nombreuses, complexes et variables en jeux vidéo, comme par exemple les jeux de rôle Donjons et Dragons (Gygax et Arneson, 1974-2014) ou les jeux de plateau Warhammer (Priestley, 1983-2010) qui posent déjà en eux-mêmes problème à de nombreuses définitions du jeu (Salen et Zimmerman, 2004, p. 82 ; Tavinor, 2008 ; Juul, 2011, p. 39). Quand des versions électroniques de ces jeux voient le jour, comme Baldur's Gate (BioWare, 1998) ou Warhammer 40,000: Chaos Gate (Random Software, 1998), il semblerait au premier abord qu'il ne s'agisse que d'une simple transposition d'un médium papier vers un médium numérique, comme c'est le cas pour les échecs, le Sudoku ou Risk. Mais la rapidité des calculs effectués par un ordinateur permet de faciliter l'accès à ces jeux, d'accélérer l'application des règles et le rythme de la partie : cette transposition semble produire une nouvelle expérience de jeu avec le même ensemble de règles.

Mon but n'est pas ici de répertorier tous les problèmes de toutes les définitions du jeu ou du jeu vidéo qui ont pu être proposées, mais l'éventail considéré suggère que ces difficultés qu'elles rencontrent ne sont pas liées qu'à leur contenu, mais aussi à la manière dont elles sont formulées. Or, comme on l'a remarqué avec Waismann, des définitions explicites peuvent être adaptées à des recherches où l'on circonscrit un domaine d'objets pour tirer des conséquences de leurs propriétés communes, comme 
en mathématiques, mais leur caractère clos et rigide les rend moins adaptées quand on cherche à caractériser un ensemble d'objets empiriques pour lequel l'application d'un terme est toujours affaire de jugement et ne peut être réduit à l'application mécanique de critères nécessaires et suffisants. Ainsi, lorsque l'on cherche à utiliser une définition explicite pour circonscrire un domaine en permanente évolution comme les jeux vidéo, celles-ci mènent irrévocablement à des problèmes de cas intermédiaires ou de cas limites comme les problèmes énoncés ci-dessus ${ }^{7}$.

On peut accepter ces difficultés et déclarer comme le fait Juul que «l'existence de cas limites n'est pas un problème pour la définition tant que l'on est capable de comprendre pourquoi et comment quelque chose est un cas limite » (Juul, 2011 [2005], p. 29). Rappelons qu'une définition explicite ne consiste qu'à résumer un definiens en introduisant un definiendum qui pourra lui être substitué à loisir: à moins que la définition ne soit contradictoire ou circulaire, on ne peut critiquer une définition explicite que sur les conséquences qu'elle implique.

Cependant, en partant d'une définition explicite, on risque de créer artificiellement des difficultés théoriques. Par exemple, si je décide de définir les baleines comme des poissons, je ne fais aucune erreur logique, mais j'aurais une définition difficile à utiliser, en contradiction avec l'usage commun de la langue et qui nécessiterait de modifier en profondeur la classification des espèces. Comme le fait remarquer le biologiste André Lwoff, « la définition est l'une des méthodes de la découverte, c'est en fait une remarquable méthode heuristique " (Lwoff, 1969, p. 11) et toutes les formes de définitions n'offrent pas les mêmes perspectives heuristiques. Les définitions explicites du jeu et du jeu vidéo semblent ainsi restreindre le champ d'étude des sciences du jeu en ne laissant pas ou peu de place à la pluralité des pratiques que l'on qualifie comme jeu dans le langage quotidien qui ne renvoient pas qu'à des systèmes de règles, mais aussi à des activités libres sans règles fixes telles qu'on les trouve chez les enfants ou les animaux (free-form play).

On peut alors se demander si les quelques tentatives de définitions non-explicites du jeu vidéo règlent les problèmes des définitions explicites et si elles offrent d'autres perspectives heuristiques.

\section{Définitions par air de famille}

41 Tavinor (2008) reconnait à raison que « les jeux vidéo sont une classe historiquement contingente et [que] toute définition utile des jeux vidéo doit admettre de potentielles futures révisions » (Tavinor, 2008). Il propose alors une « définition disjonctive » afin de réconcilier trois approches différentes du jeu vidéo comme jeu, comme narration et comme fiction narrative. Cette définition prend la forme suivante :

$42 \mathrm{X}$ est un jeu vidéo si et seulement si c'est un artefact audiovisuel, s'il est conçu principalement comme un objet de divertissement et s'il est conçu pour procurer ce divertissement par l'un des deux ou les deux modes d'engagement suivant: une jouabilité (gameplay) régi par des règles ou une fiction interactive. (Tavinor, 2008)

43 Mais malgré sa forme disjonctive, la définition qu'adopte Tavinor reste une définition explicite fondée sur une équivalence («si et seulement si »). Elle n'est pas différente de celle d'Esposito citée ci-dessus si ce n'est pour sa référence à cette notion de «divertissement» qui est pourtant controversée (voir Malaby, 2007, pp. 97-102, sur 
cette question). Il n'est alors pas étonnant que cette définition rencontre les difficultés que nous avons discutées ci-dessus.

A la différence de Tavinor, Triclot adopte clairement la notion d'air de famille pour définir les jeux vidéo à la fin du prologue de la Philosophie des jeux vidéo :

- $\mathrm{S}$ [ocrate]: Je ne pense pas qu'on puisse définir le jeu vidéo simplement par les objets que l'on utilise. On doit faire entrer en ligne de compte quelque chose d'irréductiblement subjectif. [...] Tu vois, je crois que l'on s'est trop préoccupé de savoir ce qui différenciait les jeux vidéo du cinéma, avec une réponse facile ou trop facile : l'interactivité. Et on s'est trop peu demandé ce qui distinguait les jeux vidéo des jeux au sens ordinaire. Sur quelles zones d'expérience sont-ils fondés qui n'appartiennent qu'à eux?

$-\mathrm{M}[$ ario] : C'est tout à fait mon avis.

- S.: Donc, nous pouvons dire cela : il y a dans les jeux vidéo de grands types d'expériences qui ont un air famille. Pas une seule d'ailleurs: jeux de tir, de stratégie, simulations, jeux de gestion, plateforme, jeux de rôle, etc. On ne joue pas de la même manière. Tout cela s'est bricolé au fur et à mesure de l'histoire des jeux. - M. : Des expériences avec un air de famille ? Voilà bien un critère extrêmement flou pour définir les jeux vidéo.

- S. : Parce que tu as mieux peut-être ? (Triclot, 2011, pp. 11-12)

Il est difficile de savoir si le reste de l'ouvrage de Triclot est une réponse à ce défi de Socrate ou si cette définition par air de famille fait office de définition préliminaire qui n'est pas remise en cause par la suite. Elle est, dans tous les cas, intéressante en ellemême à plus d'un titre.

D'une part elle écarte la possibilité de définir les jeux vidéo comme des artefacts et envisage plutôt de les caractériser comme des expériences. C'est ce qui amène Triclot par la suite à critiquer le programme des game studies et à y substituer des " play studies» (Triclot, 2011, p. 25) qui se concentrent sur l'activité du jeu (play), toujours protéiforme, toujours émergente, irréductible à l'analyse d'un système de règles.

D'autre part, en prenant la forme d'une définition par air de famille, elle évite le problème de Sim City que rencontrent les définitions explicites mentionnées précédemment. Si le jeu vidéo est la disjonction d'une série d'expériences (celle des jeux d'arcade, des jeux textuels, des jeux de tirs en vue subjective, des jeux de rôle, des jeux de danse et de rythme, des jeux de stratégie en temps réel, etc.), alors il n'y a aucun problème pour faire entrer, par un réseau d'analogie, Sim City, les Sims (Maxis, 2000) ou Minecraft dans la catégorie des jeux et des jeux vidéo.

On pourrait ainsi de la même manière résoudre un certain nombre de difficultés du modèle classique du jeu de Juul en ne modifiant que sa forme. Juul remarque en effet que les cas limites à son modèle peuvent être considérés comme des jeux s'ils se contentent de violer l'un de ses six critères - s'ils violent deux critères ou plus ce ne sont plus des jeux. Les simulations ouvertes comme Sim City par exemple satisfont tous les critères du modèle classique sauf celui de valorisation des résultats: elles constituent ainsi un cas limite de jeu. Par contre, le jeu de la vie de Conway (1970) n'est pas un cas limite de jeu parce qu'il ne satisfait que trois critères sur six (des règles fixes, des résultats variables et des conséquences négociables): ce n'est pas un jeu à strictement parler pour Juul (2011 [2005], p. 39).

Pour éluder le problème des cas limites et les faire entrer dans la définition des jeux de Juul, on pourrait amender la forme du modèle classique du jeu : plutôt que d'affirmer que les six critères qui le constituent sont nécessaires et suffisants, on établit qu'il est 
suffisant que cinq des six critères soient satisfaits pour qu'un objet soit qualifié de jeu. On aurait alors plus une définition explicite, mais une définition polycritérielle.

Il serait même possible d'envisager une définition aux frontières graduelles qui attribue un «score ludique " à chaque activité en fonction du nombre de critères du modèle classique du jeu qu'elle satisfait. La définition de Juul pourrait ainsi être bien plus ouverte à la pluralité des pratiques de jeu, en n'excluant plus le jeu comme activité libre sans règle (free-form play) et en suggérant des affinités intéressantes entre le jeu et d'autres activités comme la programmation, la cuisine ou la conduite automobile (Juul, 2011 [2005], p. 39).

51 La forme des définitions du jeu et du jeu vidéo est donc en partie responsable des problèmes que celles-ci rencontrent et des débats sur le champ de recherche des études du jeu qui s'en suivent. Mais si les définitions polycritérielles, par air de famille, rencontrent moins de difficultés théoriques et ouvrent plus de pistes de recherches que les définitions explicites, pourquoi ne sont-elles pas universellement adoptées? Qu'estce qui empêche Juul d'assouplir son modèle en n'exigeant pas que ses six critères soient conjoints, mais disjoints?

C'est que les définitions par air de famille s'accompagnent aussi de leur lot de problèmes. Si l'on spécifie un nombre précis de critères que doit satisfaire un objet pour que la définition s'applique, il y a toujours la question de justifier de manière non arbitraire ce nombre. Si on laisse au contraire ce nombre varier, il semble que l'on perde toute capacité à utiliser les définitions pour distinguer des ensembles ou classer des objets dans des ensembles différents.

53 Ainsi, si l'on adopte une définition par air de famille des jeux vidéo, il n'est plus possible de distinguer les jeux vidéo des jeux non vidéo. En effet, les ressemblances entre l'expérience de certains jeux vidéo et l'expérience de certains jeux non vidéo semblent plus importantes que les ressemblances qu'ont les expériences des jeux vidéo eux-mêmes. Une partie de Minecraft ou de Sim City n'a-t-elle pas plus de ressemblance avec la construction d'un château Lego ou une partie de Monopoly qu'avec le jeu de tir en première personne situé en pleine apocalypse zombie Left 4 Dead (Valve, 2008) ? En quoi l'activité d'une joueuse professionnelle de League of Legends (Riot Games, 2009) recrutée par le PSG et qui fait cinq heures d'entraînement par jour ressemble-t-elle à celle d'une joueuse qui résout un Sudoku sur son téléphone dans le bus? En quoi jouer à un jeu vidéo Warhammer 40,000: Chaos Gate serait une activité plus proche d'une partie sur la borne d'arcade Dance Dance Revolution (Konami, 1998) que d'une partie de Warhammer sur plateau suivant les mêmes règles que sa version vidéoludique?

Cette difficulté est souvent contournée en affirmant que les jeux vidéo ont une dimension virtuelle et interactive. Mais comme le fait remarquer Triclot dans le passage cité ci-dessus, on n'échappe pas ainsi au problème de la distinction entre jeu vidéo et non vidéo puisque tous les jeux ont cette dimension ${ }^{8}$.

On peut accepter cette difficulté des définitions par air de famille et y voir un signe de leur fécondité plutôt qu'un de leur problème. Une telle position est elle aussi parfaitement cohérente et n'est en aucune manière une erreur logique. Mais elle nous empêche alors de poser un certain nombre de questions (et d'y répondre), notamment celle que pose Triclot lorsqu'il affirme que l'« on s'est trop peu demandé ce qui distinguait les jeux vidéo des jeux au sens ordinaire ». Qu'est-ce que cela fait de jouer à un jeu vidéo qu'un jeu non vidéo ne nous fait pas? Y a-t-il une différence dans l'expérience de jeux qui ne pourrait exister que sous la forme de jeux vidéo et d'autres 
jeux comme le Sudoku ou le Solitaire qui ont connu des versions sur ordinateur? Autrement dit, est-il possible de donner une définition des jeux vidéo qui permette de les distinguer de leurs contreparties non vidéo tout en échappant aux difficultés des définitions explicites mentionnées plus haut?

\section{Des lois en guise de règles : proposition de définition à texture ouverte}

Pour répondre à ces questions et opérer ces distinctions, il est nécessaire de limiter partiellement l'extension du terme jeu vidéo. C'est pourquoi j'explore dans cette section la possibilité d'une définition à texture ouverte des jeux vidéo. En effet, ce type de définition a l'intérêt d'« admettre de potentielles futures révisions » comme l'exige Tavinor tout en limitant le concept de jeu vidéo «dans certaines directions" pour reprendre l'expression de Waismann.

Cependant, pour éviter que ces limitations soient trop restrictives, réduisent la pluralité des pratiques des jeux vidéo et ne reconduisent l'opposition entre le jeu défini comme artefact ou système de règles (game) et le jeu défini comme activité protéiforme ouverte et libre (play), l'enquête que j'entreprends ici vise à relier la conception du jeu vidéo comme artefact formel et matériel (c'est-à-dire comme programme numérique et algorithmique) à l'expérience qu'en font les joueuses. L'objectif est donc moins de prendre parti dans l'opposition entre game studies et play studies que d'essayer d'en sortir en utilisant les ressources de la théorie de la définition.

\section{Les règles des jeux vidéo déterminent ce qui est possible, pas ce qui est autorisé}

Qu'est-ce que cela fait de jouer à un jeu qui est un programme numérique? Tout d'abord, puisqu'un programme est un ensemble de règles, cela indique que même si tous les jeux n'ont pas forcément de règles, les jeux vidéo font partie des jeux qui ont des règles (mais nous verrons que cela ne signifie pas qu'elles se manifestent toutes de la même manière). Or, dans un jeu vidéo comme dans tout programme informatique, l'exécution des règles est prise en charge par une machine numérique automatisée : c'est une des caractéristiques les plus saillantes du jeu vidéo qui a été remarquée par l'ensemble des chercheurs traitant de ce sujet (Salen et Zimmerman, 2004, pp. 143-144; Wolf, 2008, pp. 3-4; Triclot, 2011, p. 38 ; Juul, 2011 [2005], p. 48). La définition de Karhulahti (2015) notamment repose intégralement sur cette caractéristique des jeux vidéo.

Cela a une conséquence directe: dans un jeu vidéo, il est impossible de tricher en trompant ou en achetant la vigilance de ceux qui sont censés faire appliquer cette règle puisqu'il n'y a personne qui fait appliquer les règles. Pourtant, dans son ouvrage intitulé Cheating, Consalvo (2009) étudie plusieurs cas de ce qui est considéré comme de la «triche" dans les jeux vidéo, comme utiliser des codes ou des cartouches de triche (games genies ou gameshark) ou enfreindre les règles que des communautés de joueuses s'imposent à elles-mêmes.

Mais lorsque l'on entre un code de triche (cheat code) ou une commande pour obtenir, par exemple, des vies infinies, on n'enfreint aucune règle: on découvre une règle 
cachée et on en tire parti. De la même manière, lorsque l'on trouve un défaut du logiciel (bug ou glitches) qui nous aide dans la progression du jeu, on tire partie d'une incohérence du code non prévue par les développeuses.

61 De même, lorsqu'on utilise une cartouche de triche ou un correctif (patch), on modifie le code du jeu : cela peut être considéré comme de la triche par une communauté de joueuses, mais cela consiste en réalité à jouer à une autre version des règles du jeu.

62 Enfin, lorsqu'une communauté impose certaines règles (comme ne pas utiliser tel personnage dans un jeu de combat ou ne pas utiliser tel glitche dans un jeu de course) on peut effectivement enfreindre ces règles et en ce sens tricher. Mais on parle alors d'un autre type de règle que celles qui sont contenues dans le code du jeu comme on le montre ci-dessous.

Ainsi, le fait que le jeu vidéo soit un jeu numérique implique que les règles incorporées dans le code sont absolument nécessaires et inviolables: elles ne définissent pas les actions qui sont permises ou interdites, mais les actions qui sont possibles ou impossibles.

\section{Les règles des jeux vidéo sont opaques et non publiques}

De plus, le fait que les règles du jeu vidéo soient algorithmisées (c'est-à-dire appliquées par une procédure de calcul itérative) a une conséquence supplémentaire : ces règles sont essentiellement opaques et implicites, là où les règles d'un jeu non vidéo doivent être publiques et explicites.

Sur ce point aussi, de nombreux auteurs (notamment des concepteurs de jeu comme Crawford) ont remarqué qu'il est possible aux développeuses de cacher des informations aux joueuses. C'est ainsi que Resident Evil 4 (Capcom, 2005) par exemple ne révélait rien de son système de difficulté adaptative - le nombre d'ennemis et leur force variant en fonction de la réussite des joueuses - ou que le jeu de rôle Valkyrie Profile (Tri-Ace, 1999) avait caché une variable nommée le Seal Rate dont la valeur affectait la fin du jeu'. Cette possibilité peut être déplorée, comme le fait le concepteur de jeu James Dunnigan dans l'ouvrage de Salen et Zimmerman (2004, p. 85), ou encensée, mais le caractère opaque de certaines règles des jeux vidéo semble faire consensus.

Cela montre que dans un jeu vidéo, la joueuse n'a pas à être mise au courant de toutes les règles du jeu auquel elle joue, alors que l'on ne peut pas jouer à un jeu non vidéo doté de règles sans qu'il soit au moins possible que les joueuses aient accès à ces règles publiquement et exhaustivement avant que le jeu n'ait lieu.

Certes, les développeuses choisissent souvent de nous donner un aperçu des effets que ce code a sur le jeu, à travers un livret ou un tutoriel, afin de nous indiquer comment profiter du jeu. Mais ces informations ne sont que parcellaires : si le livret de Super Mario 64 (Nintendo, 1996) explique comment effectuer un triple saut avec Mario, ce n'est que lorsque l'on a la manette en main que l'on mesure la hauteur, la longueur et la durée du saut, et donc ce qu'il va permettre ou non de faire. De plus, certains jeux vidéo, comme Metroid (Nintendo, 1986), Megaman X (Capcom, 1993) ou Shovel Knight (Yacht Club Game, 2014), se passent très bien de tels outils d'apprentissage (voir figure 1). Dans un jeu vidéo, on apprend ce que l'on peut ou ne peut pas faire en essayant, en ratant, en recommençant, c'est-à-dire en interagissant avec le jeu lui-même. 
68 n'apparaîtraient pas forcément de manière évidente. Certains jeux des premières et deuxièmes générations de console, entre autres sur Amstrad CPC-64, étaient vendus sous la forme d'un livret contenant l'intégralité du code à entrer pour pouvoir lancer le jeu. Mais le jeu qui allait résulter de l'exécution d'un tel code n'était pas pour autant prévisible pour une joueuse, sinon il aurait perdu tout intérêt en tant que jeu.

69

Dans Metroid (Nintendo, 1986), le premier item que peut acquérir l'héroïne est la Morph Ball qui lui permet de se transformer en une sphère pour rouler sous les obstacles. Aucun texte ni tutoriel n'est indispensable pour apprendre le fonctionnement de cet item : comme on le voit sur la première capture d'écran (figure 1), la joueuse qui obtient cet item est bloquée (elle ne peut ni revenir sur ses pas à droite, ni continuer à gauche), elle est donc contrainte par le design de niveau (level design) d'apprendre à utiliser la Morph Ball pour sortir de cette situation (figure 2).

Figure 1

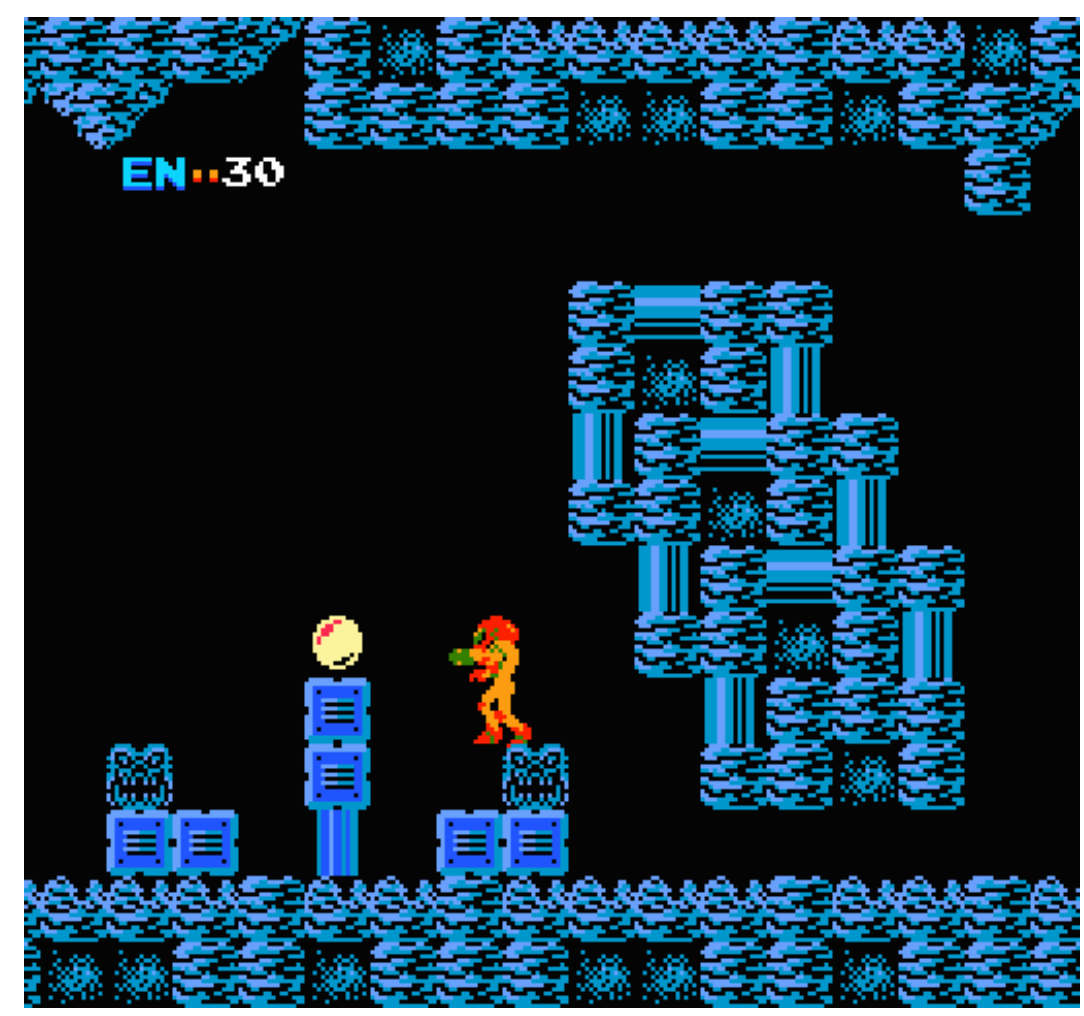




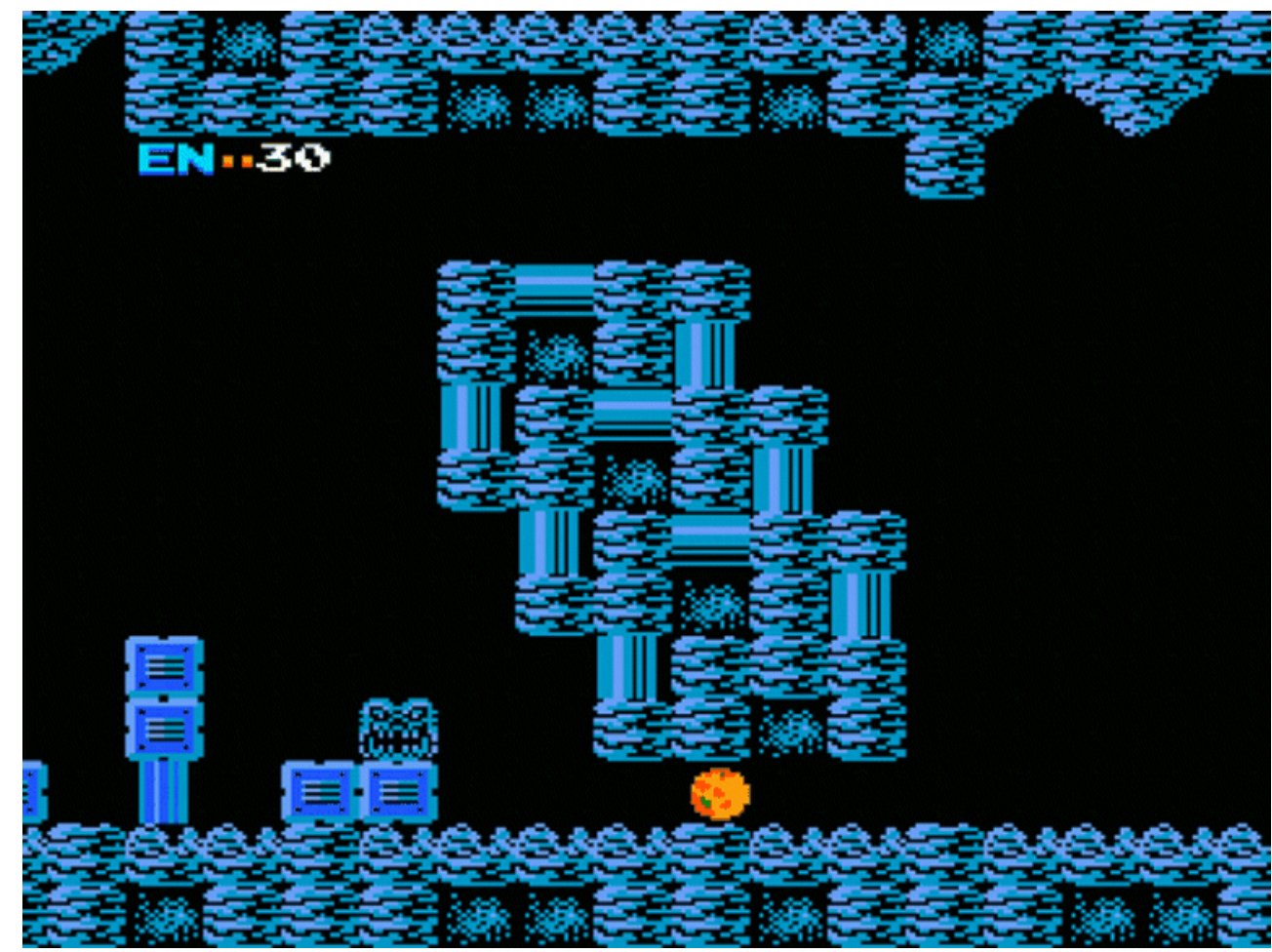

70 Les développeuses elles-mêmes n'ont pas forcément un accès transparent à tout ce qu'il est possible de faire dans leur propre jeu. Dans son étude du tournant computationnel (computational turn) en physique (c'est-à-dire des changements épistémologiques induits par la multiplication des simulations informatiques et des calculs numériques), le philosophe des sciences Humphreys a montré que l'exécution d'un programme algorithmique contient toujours une part d'opacité épistémique, c'est-à-dire une imprévisibilité du résultat due à la rapidité des calculs, à l'impossibilité de vérifier chaque étape du calcul et à l'interaction de nombreux calculs simultanés (Humphreys, 2004, pp. 147-151) ${ }^{10}$.

71 Ainsi, si l'on admet que les règles qui structurent le jeu vidéo se trouvent dans le code de son programme et sont appliquées via l'exécution de ce programme, alors cette opacité épistémique a pour conséquence que l'ensemble des actions possibles ou impossibles à faire dans un jeu n'est jamais - pour nous - parfaitement circonscrit : on peut toujours découvrir de nouvelles possibilités d'actions qui n'étaient pas prévues, pas même par les développeuses.

72 L'expérience du jeu vidéo passe donc nécessairement par des phases d'expérimentation où il ne s'agit pas de jouer selon les règles, mais de jouer pour découvrir les règles (et la meilleure manière de les exploiter). Certes, il est courant de faire des «tours pour voir » lorsqu'on joue pour la première fois à un jeu de cartes ou à un jeu de plateau - on apprend alors le contenu des règles tout en les appliquant. Mais dans de telles pratiques, il faut toujours qu'il y ait une joueuse qui connaisse déjà les règles ou qui les ait sous les yeux pour énoncer ce qui est permis et ce qui ne l'est pas. De plus, ce « tour pour voir » constitue une phase de pré-jeu, la partie ne commence que lorsqu'il est terminé. Par contre, lorsqu'on joue à un jeu vidéo, c'est le jeu lui-même qui enseigne aux joueuses ce qui leur est possible ou non de faire et cet enseignement continu tout au long du jeu représente une part importante des phases de jeu elles-mêmes ${ }^{11}$ : chaque 
nouvel item, chaque nouvel ennemi, chaque nouveau niveau, chaque nouveau boss s'accompagne son lot de règles locales qu'il faut découvrir et apprivoiser.

C'est pourquoi, comme le fait remarquer Wolf, le programme du jeu peut apparaître aux joueuses moins comme un arbitre que comme « un adversaire actif en compétition avec la joueuse humaine" (Wolf, 2008, p. 4). Cela permet d'expliquer un trait saillant qui distingue les jeux vidéo des jeux non vidéo : si la plupart des jeux non vidéo ne peuvent se jouer qu'à plusieurs, c'est l'inverse dans le cas des jeux vidéo puisque même les jeux destinés à la compétition entre humains disposent d'un mode de jeu solo. Ainsi, lorsqu'on joue à un jeu vidéo, on expérimente une forme de jeu dans lequel le dispositif matériel mis à notre disposition n'est pas qu'un médium pour affronter d'autres joueuses, mais l'objet du jeu lui-même puisqu'une part de l'activité de jeu consiste à les découvrir et à essayer de surmonter les obstacles qu'elles nous posent.

En résumé, alors que les règles d'un jeu non vidéo apparaissent aux joueuses sous une forme publique et interdisent certaines actions, les règles d'un jeu vidéo apparaissent sous une forme opaque et empêchent certaines actions ${ }^{12}$. Autrement dit, si les règles d'un jeu non vidéo se manifestent comme des énoncés déontiques (comme des lois politiques) qu'il s'agit d'appliquer, les règles d'un jeu vidéo se manifestent comme des énoncés nomologiques (comme des lois de la nature) qu'il s'agit de découvrir par l'expérimentation ${ }^{13}$.

Cependant, cette affirmation demande à être précisée pour être correcte. Dans les jeux vidéo comme ailleurs, il peut exister des énoncés déontiques qui se superposent aux énoncés nomologiques : c'est le cas lorsque des communautés de joueuses se donnent des règles prohibant certaines actions et prévoient des sanctions pour ceux qui les enfreignent (être banni d'une guilde de jeu en ligne par exemple). Ces règles peuvent prendre une dimension particulièrement importante dans les jeux multijoueurs qui impliquent une grande part d'interaction entre joueuses. Comme on l'a vu, ces règles se comportent comme des règles de jeux non vidéo et on peut tricher en essayant de les enfreindre sans que cela soit remarqué. Mais on peut distinguer les jeux non vidéo et les jeux vidéo en ce que les premiers ne sont constitués que d'énoncés déontiques, tandis que les seconds impliquent aussi des énoncés nomologiques.

Ainsi, une partie des règles du jeu vidéo - celles qui sont inscrites dans le code du programme - structurent un monde comme les lois de la nature structurent un univers, c'est-à-dire en rendant impossibles certains événements et en laissant possibles d'autres. C'est à la joueuse de découvrir ces lois et ces possibilités qui découlent de l'exécution du programme. Cette phase de découverte des règles fait partie intégrante du jeu vidéo. La définition à texture ouverte du jeu vidéo comme expérience spécifique que je propose ici est donc la suivante :

77 Si un jeu est un jeu vidéo, alors la découverte de ses règles fait partie de l'activité de jeu et une partie de ses règles au moins se manifestent aux joueuses comme des énoncés nomologiques (lois de la nature) et non déontiques (lois politiques).

78 Puisqu'il s'agit d'une définition ayant la forme d'un concept à texture ouverte, il y a la possibilité de rajouter une ou plusieurs conditions à celles mentionnées ici. Dans la section suivante, nous allons voir s'il est nécessaire de compléter cette définition pour la clarifier. 


\section{Conséquences et clarifications}

\section{Intérêt d'un concept à texture ouverte du jeu vidéo}

1. Les définitions explicites fondées sur la notion de conflit, de performance ou d'objectif ont du mal à rendre compte de jeu de type bac à sable comme Sim City ou Minecraft, des jeux sans buts apparents qu'elles rapprochent plus du jouet (comme les Lego) que de l'authentique jeu. Mais si l'on définit l'expérience de jeu vidéo comme une expérimentation avant d'être une compétition visant l'amélioration des performances, on a moins de mal à inclure ces jeux dans la catégorie des jeux, parce que le plaisir de l'expérience de jeu ne réside pas uniquement dans la victoire, mais aussi dans la découverte pure et simple du monde vidéoludique et de ses règles. Les jeux de type bac à sable ne représentent pas alors un cas limite, mais au contraire un cas paradigmatique de l'expérience jeu vidéo : dans de tels jeux, les règles n'ont pas besoin d'être finalisées par un but, un objectif à atteindre, elles existent uniquement comme des lois, des contraintes qui délimitent un espace d'actions possibles. Ainsi, jouer à Minecraft ou à tout autre jeu vidéo de type bac à sable diffère radicalement de l'expérience de jeu avec des jouets comme les Lego parce que Minecraft dispose d'un système de mélange de matériaux (crafting) qui invite la joueuse à l'expérimentation et ne pourrait exister sans l'aide d'un programme gérant algorithmiquement le résultat des différents mélanges.

81 2. Quant au problème de la transposition de jeux comme Risk, le Sudoku ou les échecs sur des supports numériques, on peut remarquer que nos réticences à considérer que jouer à Risksur console, au Sudoku sur un smartphone ou aux échecs sur un ordinateur est une expérience de jeu vidéo viennent du fait qu'il y a peu de phases d'expérimentation avec les règles. Peut-être va-t-on essayer quelques commandes spécifiques que l'on ne pourrait pas faire sur un plateau, sur papier ou sur un échiquier classique, comme visualiser les différents coups possibles ou se faire aider d'un indice fourni par le programme : cela suffit pour distinguer la version vidéo et non vidéo de ces jeux, mais pas pour considérer qu'il y a là une expérience qui ne pourrait être proposée que par un jeu vidéo. On peut reprendre la terminologie de Triclot (2011, p. 11) de «jeux sur ordinateur » plutôt que de jeu vidéo pour nommer cette catégorie de jeu.

82 3. Le problème posé par les jeux de rôle comme Donjons et Dragons ou Warhammer est différent. Ce sont bien les mêmes règles qui gouvernent la version plateau et la version vidéo du jeu, mais la prise en charge de leur application par un automate algorithmique produit une expérience de jeu profondément différente. D'une part, toute une phase de préparation des figurines (achat, peinture, disposition sur le plateau, etc.) disparaît dans la version vidéo et d'autre part la rapidité des calculs numériques donne au jeu un autre rythme. Cependant, la dimension d'exploration et d'expérimentation avec les règles ne prend une part déterminante dans cette expérience de jeu que lorsque la joueuse décide de jouer seule à ces jeux ou lorsque la règle selon laquelle le jeu se déroule tour par tour est remplacée par des actions en temps réel (on passe alors d'un jeu de stratégie au tour par tour à un jeu de stratégie en temps réel, forme de jeu vidéo qui n'a pas de contrepartie parmi les jeux sur plateau). 
83 Ainsi, si l'on définit le jeu vidéo comme un concept à texture ouverte, on conserve la possibilité de distinguer la pratique vidéoludique d'autres formes de jeu sans pour autant clore la définition du jeu vidéo en réduisant la diversité des jeux ou en empêchant toute révision ultérieure de ce concept. De plus, la définition proposée dans la section précédente permet d'attirer l'attention sur des objets d'étude mis à la marge jusqu'ici, comme les phases de prise en main des jeux durant lesquelles la joueuse découvre elle-même par essai et erreur les règles du jeu auquel elle joue. Ces moments de jeu diffèrent des phases d'entraînement dans les jeux non vidéo parce qu'elles représentent une expérimentation plus qu'une amélioration des résultats. L'hypothèse qui découle de cette conception est que ces phases de prise en main ne cessent réellement jamais et que la joueuse est perpétuellement en train d'apprendre les règles. La définition des jeux vidéo comme jeux dans lesquels les règles se manifestent comme des lois qu'il faut découvrir plutôt que respecter permet donc de répondre à un certain nombre de problèmes des définitions classiques des jeux vidéo et d'ouvrir des pistes de recherches fécondes. Mais ne rencontre-t-elle pas elle-même d'autres problèmes liés à sa forme?

\section{Problèmes des concepts à texture ouverte}

Cette définition pourrait décevoir ceux qui recherchent une essence du jeu vidéo, ce qui serait au cœur de l'expérience vidéoludique, une part de l'expérience du jeu vidéo qui générerait le plaisir spécifique de jouer aux jeux vidéo. En effet, une définition à texture ouverte n'est pas une définition essentialiste : elle ne vise pas à fournir ce qu'il y a de commun à tous les jeux vidéo ni à être exhaustive ni même à trouver ce qui produit causalement tous les aspects de l'expérience du jeu vidéo.

86 Ainsi, si l'on impose comme condition minimale à un jeu vidéo d'être un jeu où les règles sont expérimentées et découvertes, il est clair que cette dimension d'expérimentation est plus centrale dans l'expérience des jeux d'exploration, d'aventure ou d'énigmes comme Portal (Valve, 2007), qui se jouent seul, qu'à des jeux multijoueurs comme Starcraft (Blizzard, 1998) ou Street Fighter 2 (Capcom, 1992) dans lesquels la dimension de compétition est importante: ces types d'expériences vidéoludiques demanderaient probablement que l'on complète la définition proposée ici pour limiter dans une nouvelle direction le concept à texture ouverte de jeu vidéo. De même, lorsque l'on joue à un jeu de tir sur rails (rail shooter) comme Time Crisis (Namco, 1995) ou à un jeu de danse comme Dance Dance Revolution, le fait de découvrir les règles est une part bien moins importante de l'expérience de jeu que la maîtrise progressive de l'habileté nécessaire pour progresser dans les niveaux.

Cependant, même dans Starcraft ou dans Street Fighter les phases d'entraînement des joueuses continuent de comporter une part de découverte et d'expérimentation des règles : il s'agit de voir ce que l'on peut faire avec telle unité ou tel personnage, c'est-àdire ce que le programme du jeu nous laisse faire. De même, dans un jeu comme Time Crisis, il faut apprendre certaines règles du jeu par l'expérimentation (par exemple que c'est en se mettant à couvert que l'on recharge) et l'on découvre à chaque niveau les nouvelles règles qui accompagnent les nouveaux ennemis, les nouvelles armes, etc. Enfin, même dans un jeu de rythme comme Dance Dance Revolution, la maîtrise du jeu passe aussi par la découverte de la manière dont fonctionne le système de score, le déroulement des instructions à l'écran, les différences entre niveaux de difficulté, etc. 
La preuve que cette dimension d'expérimentation avec les règles est présente dans ces jeux (même si elle ne constitue pas le cœur de l'expérience ludique) est que l'on peut très bien se mettre à jouer à ces jeux sans avoir pris connaissance d'aucune de leurs règles, comme c'était le cas dans les salles d'arcades où les premières bornes de Time Crisis et Dance Dance Revolution ont été mises sur le marché. Ainsi, ces cas satisfont la définition a minima du jeu vidéo comme un jeu où l'on peut apprendre les règles sans qu'elles soient énoncées.

Un autre volet d'objections consiste à dire qu'une définition à texture ouverte est trop libérale parce qu'il existe de nombreux jeux non vidéo qui satisfont les critères nécessaires proposés ici. Mais puisque ces critères sont nécessaires et non suffisants, alors il n'y a aucune incohérence à ce que des jeux non vidéo les satisfassent également. On peut même considérer que c'est l'une des forces de ce type de définition que de suggérer des rapprochements surprenants avec des activités habituellement considérées comme sans parenté avec le jeu vidéo. Je vais considérer dans ce qui suit deux cas qui me semblent ouvrir des pistes de recherche pertinentes.

1. Le premier cas est celui de la recherche scientifique. Comme l'affirmait Lwoff dans son discours de réception du prix Nobel : "c'est la recherche qui m'a conduit ici et la recherche, on le sait, est un jeu» (Lwoff, 1965). Or c'est un jeu dont l'objet est la découverte et l'expérimentation avec des énoncés nomologiques: les lois physiques, biologiques, économiques, sociales, etc., de notre univers. Elle satisfait donc le concept à texture ouverte de jeu vidéo proposé plus haut.

On pourrait ajouter une nouvelle condition nécessaire à ce concept pour mieux le délimiter et le distinguer de la recherche scientifique (par exemple que les énoncés nomologiques avec lesquels expérimentent les joueuses aient été produits par d'autres êtres humains), mais en laissant ouverte la définition du jeu vidéo on révèle des liens inattendus entre jeu et recherche. D'une part, la proximité entre certains jeux vidéo de type bac à sable, comme Universe Sandox (2012, Giant Army), et des simulations numériques utilisées en astrophysique, comme le Millennium Simulation Project, renseigne sur la manière dont les jeux vidéo (depuis Spacewar! [Russell, 1962] qui avait été programmé sur des ordinateurs destinés à des calculs de balistique) se sont développés conjointement aux efforts pour modéliser et simuler des systèmes physiques. Et d'autre part, cela suggère que le plaisir de jouer aux jeux vidéo est apparenté au plaisir de la découverte scientifique. C'est sur cette idée Raph Koster fonde sa Theory of Fun qui voit dans jeu un «instrument fondamental et puissant d'apprentissage » (Koster, 2005, p. 37) ${ }^{14}$.

91 Mais, à la différence de ce qu'affirme Koster, je soutiens que cette compréhension a une dimension tout autant (voire plus) corporelle et gestuelle qu'intellectuelle. C'est ce que révèle un deuxième rapprochement entre les jeux vidéo et une autre activité : les activités physiques comme le sport.

2. Tous les jeux qui consistent en une activité physique ou sportive satisfont la définition proposée ci-dessus : les jeux physiques sont toujours aussi des jeux avec la physique, parce qu'ils demandent d'interagir avec des objets physiques (comment le ballon rebondit, comment la raquette entre en contact avec la balle, comment mes semelles ou mes pieds adhèrent au sol, etc.) et parce que ces interactions nous font découvrir les lois physiques de notre univers (loi des chocs, loi de la gravitation, effets des frottements, conservation du moment cinétique, etc.). 
93 À nouveau, il serait possible de limiter la définition à texture ouverte que l'on a donné des jeux vidéo pour exclure les sports de cette définition. Par exemple, on peut remarquer que dès qu'une activité sportive s'organise en compétition il est nécessaire d'établir des règles déontiques : si je peux apprendre à mettre un ballon dans un arceau par essais et erreurs, en expérimentant les lois naturelles de notre univers, je ne pourrai jamais apprendre ainsi qu'un panier de basket vaut deux ou trois points selon la distance à laquelle je tire.

94 Une seconde option est d'accepter qu'il existe un lien profond entre l'expérience de jeu vidéo et la dimension non-réglée des activités physiques et sportives, lien qui n'est pas épuisé par les adaptations de sports en jeux vidéo comme le propose la série des FIFA (EA Sport, 1993 à aujourd'hui) pour le football. Une piste de recherche féconde permettrait non seulement de comprendre comment un champ de la pratique du jeu vidéo (le sport électronique ou e-sport) s'est constitué en adoptant la structure des compétitions sportives, mais aussi de rejeter le préjugé considérant le jeu vidéo comme une forme de jeu où le corps des joueuses n'est que peu engagé. Une des joueuses qu'interroge Roustan décrit ainsi la jouissance qui peut exister dans l'apprentissage d'habitudes corporelles pour un jeu vidéo :

Si c'est un jeu que j'ai envie de faire, je vais passer le temps qu'il faut pour me rappeler que dans ce jeu-là c'est telle ou telle touche, et apprendre aux doigts, parce que les doigts font les gestes automatiquement. Donc je vais apprendre que c'est ZWX. C'est ça qu'est rigolo aussi, c'est que quand t'y arrives, t'oublies ça. Quand tu fais que regarder l'écran et que tes mains elles ont appris. (Roustan, 2003, pp. 43-44)

Enfin, cela permet de relier le jeu vidéo à tous les jeux qui consistent uniquement à interagir avec un environnement physique sans but ni règle préétablis, uniquement pour le plaisir que procurent les interactions que l'on peut avoir avec cet environnement. On peut alors revenir sur la distinction classique entre play et game: il y a une parenté entre les enfants qui jouent avec des mobiles, les chats qui jouent avec des insectes ou les loutres qui jouent avec des cailloux d'un côté et les joueuses qui, dans les jeux vidéo, décident de "ne pas suivre l'histoire ", d'" essayer juste de voir ce que l'on peut faire ", ou « d'appuyer sur tous les boutons ». Pourtant, les premiers sont souvent pris comme exemples d'activités ludiques sans règle alors que les jeux vidéo semblent représenter le paradigme même du jeu comme système de règles. Cela suggère que l'émergence du jeu vidéo ne vient pas renforcer la distinction entre game et play (si elle a jamais été pertinente), mais la dépasser ou, du moins, la brouiller.

En définitive, une définition à texture ouverte est une définition autant intéressante par les limites qu'elle impose que par celles qu'elle n'impose pas et les continuités, parfois surprenantes, qu'elle suggère entre le jeu vidéo et d'autres formes d'activités ludiques. Mais si l'on définit les jeux vidéo comme des jeux qui impliquent une phase d'expérimentation avec des règles qui se manifestent comme des lois de la nature, il est naturel de se demander quelle réalité modélisent ces règles : une version appauvrie des lois de notre univers ou d'autres mondes possibles structurés par des lois nouvelles et différentes? 


\section{Jouer dans d'autres mondes possibles}

98 J'ai soutenu dans les sections précédentes que l'expérience du jeu vidéo comprend nécessairement le fait que les règles d'un jeu vidéo apparaissent aux joueuses comme des lois de la nature qu'il s'agit de découvrir par l'exploration et l'expérimentation. Je prolonge ici cet argument en montrant que le plaisir que l'on tire de cette expérience peut venir de la découverte d'autres systèmes de lois que celles qui régissent notre univers, c'est-à-dire d'autres mondes possibles.

Il paraît évident que les règles des jeux vidéo ne sont pas de simples répliques des lois physiques, sociales ou psychologiques de notre univers. La chute des pièces, dans Tetris, se fait à vitesse constante, et non à accélération constante comme le voudrait la loi de la chute des corps ; Mario, dans Super Mario Bros. (Nintendo, 1985), ne connaît pas la loi d'addition des vitesses : lorsqu'il saute d'une plateforme en mouvement, la vitesse de celle-ci ne lui est pas communiquée ; et le Gandhi de Civilization (Micropose, 1991) se transforme rapidement en un chef d'Etat belliciste prompt à employer l'arme nucléaire.

Néanmoins, l'histoire des jeux vidéo ne présente-t-elle pas une tendance constante vers le réalisme, vers des graphismes et des moteurs physiques toujours plus proches de la réalité ? Si les règles des jeux vidéo sont différentes de celles de notre univers, n'est-ce pas uniquement à cause de certaines limitations techniques ou pour simplifier la prise en main de ces jeux ? Ainsi, les pièces de Tetris tombent à vitesse constante parce que sinon elles ne laisseraient que trop peu de temps aux joueuses pour réagir ; Mario ne respecte pas loi d'addition des vitesses parce que sinon il serait trop complexe de prédire où ses sauts le feraient atterrir ; quant au Gandhi de Civilization, son agressivité n'est due qu'à une malencontreuse erreur de programmation.

101 En ce sens, les jeux vidéo ne nous présenteraient pas d'autres mondes possibles, structurés par des lois différentes du nôtre, mais uniquement des versions simplifiées de notre réalité, simplifiées pour que l'on puisse prendre plaisir à y jouer. Steven Poole, dans Trigger Happy est l'un de ceux qui a le plus clairement exposé et soutenu cette thèse :

102 En général, la philosophie qui sous-tend la manière dont les jeux vidéo construisent des mondes est que certains aspects de la réalité peuvent être modelés de manière réaliste, tandis que d'autres sont délibérément biaisés, leurs effets sont exagérés ou atténués selon les exigences du jeu. (Poole, 2004, p. 89)

Poole soutient cette thèse en argumentant que notre plaisir à jouer à un jeu vidéo provient de notre capacité naturelle à manipuler des objets dont la dynamique est similaire à celle de notre expérience quotidienne :

Nous avons l'habitude de manipuler des objets qui ont une masse, une vélocité et qui rebondissent dans le monde réel et nous pouvons prédire plutôt bien nos interactions quotidiennes avec eux. [...] L'appréciation des propriétés dynamiques est câblée [hardwired] dans notre espèce - elle est essentielle à la survie. C'est l'une des voies les plus simples par laquelle les jeux vidéo nous parlent du monde réel, en s'adressant directement à notre cerveau animal et viscéral. (Poole, 2004, p. 92-93)

Il poursuit son raisonnement en faisant remarquer que plus un jeu est modélisé de manière réaliste, plus «cela nous permet d'exercer avec plaisir nos intuitions physiques » (Poole, 2004, p. 93). Son argument est donc le suivant: les jeux vidéo ne nous fournissent pas d'autres réalités que la nôtre, ils ne nous fournissent qu'une 
version simplifiée de notre réalité, parce que nous avons tous une physique naturelle innée (" câblée » dans notre cerveau via l'évolution) qui implique que nous prenions plus de plaisir avec des objets dont la physique ressemble à celle de ceux de nos objets quotidiens. Les quelques entorses faites à la réalité ne sont dues qu'aux limites technologiques ou à la nécessité d'offrir une expérience de jeu suffisamment simple pour être plaisante.

Cette thèse est attrayante parce qu'elle semble s'appuyer sur les travaux de psychologie cognitive étudiant la physique naïve ${ }^{15}$ pour nous attribuer une prédisposition innée que nous partagerions avec d'autres animaux à saisir les lois physiques ${ }^{16}$ et pour remettre en question la distinction traditionnelle entre le monde réel de l'expérience quotidienne et le monde virtuel de l'expérience vidéoludique. Cependant, elle doit affronter (au moins) deux objections majeures. D'une part, si nous avons bien une physique naïve innée que nous aurions acquise au cours de l'évolution des espèces, celle-ci devrait être adaptée à la physique des objets tels qu'ils se comportent dans l'expérience quotidienne. Or, toute une catégorie de jeux vidéo - qui sont pourtant réalistes au sens où ils implémentent des règles qui reproduisent des lois physiques telles que la loi de la gravitation sans les déformer - nous propose de prendre plaisir avec des objets dont le comportement diffère profondément des objets de la vie quotidienne : les jeux vidéo qui prennent place dans l'espace. Spacewar! lui-même, un des premiers jeux vidéo, programmé par des étudiants du MIT sur des ordinateurs DEC PDP-1 au début des années 1960, propose un combat spatial entre deux vaisseaux dotés d'une inertie autour d'une étoile ayant un champ gravitationnel qui décroit avec le carré de la distance. Une grande partie du plaisir de jeu vient du fait que le déplacement des vaisseaux demande de s'adapter à une physique qui, quoique réaliste, est loin d'être intuitive: sans frottement les vaisseaux conservent leur vitesse et les trajectoires ne sont que très peu affectées par les commandes des joueuses. On comprend mal comment un tel exemple pourrait être expliqué par la thèse de Poole.

107 La deuxième objection que l'on peut faire à la thèse de Poole est que les jeux vidéo ne font pas que simplifier les lois de notre réalité : ils en rajoutent de nouvelles. Dans un jeu vidéo, une règle devient une loi, donc tous les objets qui sont définis par les règles du programme du jeu (y compris les menus, les fonctions de pause, de mort, de sauvegarde ou les items) obéissent à des lois naturelles, dont certaines n'ont aucune contrepartie dans le «monde réel » de notre expérience quotidienne. C'est ce que Poole lui-même semble remarquer lorsqu'il constate que

par leurs effets instantanés et magiques, les bonus (power-ups) participent d'une ontologie différente de tout autre objet à l'écran. Leur mode d'existence et leurs effets sont purement relationnels et redéfinissent la logique d'interaction entre le personnage du joueur et des ennemis. (Poole, 2004, p. 109)

C'est pourquoi l'on ne trouve pas que des physiques appauvries dans les jeux vidéo, mais également des physiques enrichies. Dans Portal par exemple, la joueuse a la capacité d'ouvrir des portails entre deux lieux éloignés pour se transférer immédiatement de l'un à l'autre. Combinée à une autre loi physique, celle de la conservation du moment cinétique (qui est, elle, une loi "réaliste» au sens de Poole), cette nouvelle loi de la physique permet d'interagir avec des objets, de produire des effets et de résoudre des énigmes d'une manière innovante et ludique précisément parce que l'on n'y a pas accès dans le monde réel. De même, dans The Legend of Zelda: Breath of the Wild (Nintendo, 2017), il est possible de combiner la loi de conservation du moment cinétique avec une 
loi de la nature inédite : certains objets peuvent être figés dans le temps et accumuler l'énergie des chocs qu'ils reçoivent jusqu'à la convertir instantanément en vitesse.

Ces exemples montrent que, comme l'avait fait remarquer Aarseth : « les jeux ne sont pas des fictions, mais un différent type de monde situé entre les fictions et notre monde : le virtuel » (Aarseth, 2007, p. 36). En effet, les jeux vidéo sont tout autant des sous-simulations (des simulations imparfaites de la réalité) que des sur-simulations, c'est-à-dire des simulations de mondes dans lesquels il est possible de faire varier des règles qui, dans notre univers, restent fixes. Ils ne sont pas les seuls à proposer ainsi des univers qui obéissent à d'autres règles que les nôtres : la bande dessinée, le cinéma, la littérature fantastique dépeignent de tels mondes depuis plusieurs siècles l'appellation de " physique de dessin animé » (cartoon physics) ayant même été inventée pour décrire les créations des dessinsanimés de Tex Avery, Hanna Barbera et de la Warner en la matière. Mais les jeux vidéo ne font pas que représenter de nouvelles physiques: ils nous permettent d'interagir avec elles et ainsi démontrent notre capacité à comprendre, agir et manipuler des objets dans des mondes différents du nôtre.

110 L'erreur de Poole est d'avoir mal interprété les travaux de psychologie cognitive portant sur la physique naïve : d'après leurs résultats, ce ne sont pas les lois de la nature elles-mêmes qui sont câblées dans notre esprit, comme s'il s'agissait d'idées innées cartésiennes. Ce sont les dispositions pour acquérir une connaissance infrathéorique, corporelle, de ces lois qui sont innées ${ }^{17}$. Le plaisir de jouer ne vient pas que $\mathrm{du}$ fait d'exercer les intuitions physiques provenant d'une physique naïve déjà acquise, mais aussi d'exercer à nouveau, dans un cadre renouvelé, ces capacités qui nous ont permis de découvrir et d'acquérir ces intuitions physiques. Le plaisir de jouer à Street Fighter 2 ne vient pas du fait que les boules de feu ont une inertie comme des cailloux que l'on s'amuse à jeter dans l'eau: il vient du fait d'apprendre la physique de ces nouveaux objets comme nous avons appris celle de notre univers en faisant des ricochets.

\section{Conclusion : ludification et narration}

111 Dans cet article, j'ai soutenu qu'un certain nombre de difficultés liées aux définitions des jeux et des jeux vidéo en particulier vient de la forme de ces définitions. Sur ce sujet, l'apport principal de la théorie de la définition qui a été développée en philosophie et en logique est de questionner le paradigme de la définition explicite en montrant que ce type de définition n'est ni le seul ni le plus adapté aux concepts empiriques.

112 J'ai exposé la possibilité d'une définition à texture ouverte des jeux vidéo reposant sur l'idée qu'un critère nécessaire pour parler d'expérience vidéoludique est de prendre en compte la manière dont les règles de ce jeu se manifestent aux joueuses. J'ai exploré les conséquences théoriques de cette définition en montrant que bien loin de réduire la pluralité des jeux vidéo a un modèle unique, elle suggère des continuités avec d'autres pratiques comme la recherche scientifique et l'activité physique.

113 Cette définition a par ailleurs une conséquence pratique, et non théorique, concernant la ludification d'activités comme l'enseignement. La définition proposée ici des jeux vidéo se démarque profondément de celles qui considèrent qu'un jeu est 
essentiellement un système permettant d'organiser un conflit et d'évaluer des résultats, comme celle de Karhulahti qui considère les jeux vidéo comme des « artefacts qui évaluent nos performances ». Dans le cadre d'une telle définition, une enseignante cherchant à ludifier l'apprentissage d'une leçon peut par exemple se contenter de proposer un questionnaire à choix multiples en ligne qui évalue automatiquement les réponses des élèves: un tel dispositif correspond parfaitement à la définition de Karhulahti, mais ne propose en définitive qu'une version numérique de la classique interrogation écrite. Il n'y a là qu'une ludification de façade : le passage par un artefact qui évalue les performances n'est que le déguisement ayant les atours d'un jeu pour mieux reconduire un dispositif d'examen.

D'après la définition que l'on a défendue, un tel questionnaire informatisé n'est pas un jeu vidéo parce que le programme utilisé ne fournit pas d'expérience ludique au cours de laquelle la découverte des règles fait partie intégrante du jeu. Pour ludifier une leçon, une enseignante ne se contentera pas de proposer une évaluation automatisée à ses élèves : elle pourra par contre transformer la leçon elle-même en jeu vidéo dans lequel les élèves apprennent en expérimentant et produisent par elles-mêmes les contenus pédagogiques ${ }^{18}$.

En définitive, le principal aspect que la définition à texture ouverte du jeu vidéo semble oublier est une dimension que des auteurs comme Juul, Esposito ou Tavinor soulignent d'emblée : un jeu vidéo peut raconter une histoire. Cela signifie-t-il que l'aspect narratif du jeu vidéo doit être tenu pour inessentiel et second ? Doit-on décréter que des jeux comme les histoires interactives (visual novel) ne sont pas réellement des jeux vidéo ? La question est débattue, mais si l'on veut faire une place à ces fictions interactives il serait effectivement nécessaire d'introduire une disjonction dans la définition du jeu vidéo comme le suggère Tavinor.

\section{BIBLIOGRAPHIE}

AARSETH E. (2007), « Doors and Perception : Fiction vs. Simulation in Games », Intermédialités, 9, pp. 35-44.

BETH E. W. (1962), « Observations concernant la théorie de la définition », in Annales scientifiques de l'Université de Clermont-Ferrand 2, tome 7, série Mathématiques $\mathrm{n}^{\circ} 1$, Clermont-Ferrand.

CAILLOIS R. (1967 [1958]), Les jeux et les hommes : le masque et le vertige, Paris, Gallimard. CONSALVO M. (2009), Cheating: Gaining Advantage in Videogames, Cambridge, MIT Press.

CRAWFORD C. (1982), The Art of Computer Game Design, McGraw-Hill.

DEMAZEUX S. (2013), Qu'est-ce que le DSM ? Genèse et transformations de la bible américaine de la psychiatrie, Paris, Ithaque.

DOWEK G. (2011), Les Métamorphoses du calcul. Une étonnante histoire des mathématiques, Paris, Le Pommier. 
ESPOSITO N. (2005), « A short and simple definition of what a videogame is », in Proceedings of DiGRA 2005 Conference : Changing Views - Worlds in Play

GARCIN B. (2014), « KerbalSpace Program, le jeu vidéo qui permet d'apprendre ses cours de physique »

GOPNIK A. et WELLMAN H. (2012), « Reconstructing constructivism : Causal models, Bayesian learning mechanisms, and the theory theory, », Psychological Bulletin, 138, pp. 1085-1128.

HOBBES T. (2000 [1651]), Leviathan, Paris, Gallimard.

HUIZINGA J. (1988 [1938]), Homo ludens, essai sur la fonction sociale du jeu, Paris, Gallimard.

HUMPHREYS P. (2004), Extending Ourselves : Computational Science, Empiricism, and Scientific Method, Oxford, Oxford University Press.

JUUL J. (2011 [2005]), Half-Real, Video Games Between Rules and Fictional Worlds, Cambridge, MIT Press.

KARHULAHTI V. (2015), « Defining videogames », Game Studies, 15

KOSTER R. (2005), Theory of Fun for Game Design, O’Reilly Media, Sebastopol.

LECONTE G. (2014), « Virtualité et interactivité du jeu vidéo », in S.Coavoux, H. Ter Minassian et S.Rufat (dir.), Espaces et temps dans les jeux vidéos, Questions Théoriques, Paris, pp. 51-72.

LWOFF A. (1965), « André Lwoff's speech at the Nobel Banquet in Stockholm, December 10, 1965 » LWOFF A. (1969), L'Ordre biologique, Paris, Robert Laffont.

MALABY T. M. (2007), « Beyond Play, A New Approach to Games », Games and Culture, 2, pp. 95-113 MONK J. D. (1976), Mathematical Logic, New York, Springer Verlag.

PADOA A. (1901), « Essai d'une théorie algébrique des nombres entiers, précédé d'une introduction logique à une théorie déductive quelconque ", in Bibliothèque du Congrès International de Philosophie, vol III, Paris.

POOLE S. (2004), Trigger Happy: Videogames and the Entertainment Revolution,New York, Arcade.

RIVENC, F. (1992), Logique et fondements des mathématiques, Anthologie (1850-1914), Paris, Payot. ROUSTAN Mélanie (2003), La Pratique du jeu video : réalité ou virtualité ?, Paris, L'Harmattan. SALEN K. et ZIMMERMAN E. (2004), Rules of Play, Game Design Fundamentals, Cambrdige, MIT Press. SMITH, B. et CASATI R. (1994), « Naivephysics : an essay in ontology », Philosophical Psychology, 7, pp. 227-247.

SPELKE E. (2007), « Innéisme, liberté et langue », in J. Brimont et J. Franck (dir.), Cahier Chomsky, Paris, L'Herne.

SUITS B. (1978), The Grasshopper : Games, Life and Utopia, Toronto, The University of Toronto Press. SUMMERS N., « Resident Evil 4 secretly adjusted its difficulty for you » SUPPES P. (1957), Introduction to Logic, New York, Van Nostrand Reinhold Company.

TAVINOR G. (2008), « Definition of videogames », Contemporary Aesthetics, 6

TRICLOT M. (2011), Philosophie des jeux vidéo, Paris, Zones.

WAGNER, P. (2017), Définition (A) 
WAISMANN, F. (2004), « La Vérifiabilité », in Laugier S. et Wagner P. (dir.), Philosophie des sciences : Expériences, théories et méthodes, Paris, Vrin, pp. 325-360.

WITTGENSTEIN L. (1961), Tractatus logico-philosophicus suivi de Investigations philosophiques, traduction française Pierre Klossowski, Paris, Gallimard.

WOLF M.(2008), The Video Game Explosion : A History from PONG to PlayStation and Beyond, Greenwood Press, Westport.

ZABBAN V. (2013) ; « Retour sur les gamesstudies. Comprendre et dépasser les approches formelles et culturelles du jeu vidéo », Réseaux, 20, pp. 139-176.

\section{NOTES}

1. J'utilise, dans cet article, le féminin comme genre par défaut.

2. Pour une recontextualisation du débat sur la définition du jeu vidéo dans le cadre de l'élaboration d'études autonomes sur le jeu vidéo, voir Zabban (2013, pp. 143-150).

3. Pour une présentation plus complète et générale de ces recherches au croisement de la logique et de la philosophie qui constituent la théorie de la définition, voir Wagner (2017).

4. Voir Monk (1976, pp. 365-366) pour une preuve du théorème de Beth qui expose ces conditions. 5. Sur ce manuel et les difficultés épistémologiques et éthiques qu'il soulève, voir Demazeux (2013). On montre plus loin que certaines définitions des jeux vidéo, bien qu'elles soient présentées comme des définitions explicites sont en réalité polycritérielles.

6. Dans une certaine mesure, Triclot accomplit le même geste lorsqu'il considère que « les jeux vidéo bousculent les règles de la syntaxe de Caillois" et "transgressent les associations habituelles des jeux traditionnels sur deux points essentiels qui correspondent à deux innovations ludiques monumentales: l'ouverture d'une zone agôn-ilinx-ludus d'une part, l'invention d'une combinaison inédite de simulation et de calcul d'autre part » (Triclot, 2012, p. 52). Mais nous verrons plus loin que la position de Triclot est différente puisqu'elle ne repose pas sur une définition explicite du jeu ni du jeu vidéo.

7. Même un auteur comme Malaby, qui se refuse à intégrer dans sa conception du jeu le fait que celui-ci soit une activité séparée en le définissant comme des domaines partiellement délimités et socialement légitimes d'une contingence contrainte qui génère des résultats interprétables » (Malaby, 2007, p. 96), use lui aussi d'une définition explicite qui est, dans sa forme sinon dans son contenu, close.

8. Voir aussi Leconte (2014) qui soutient que l'interactivité et la virtualité du jeu vidéo sont des obstacles épistémologiques pour comprendre la spécificité de cet objet.

9. Voir Summers (2015) et https://strategywiki.org/wiki/Valkyrie_Profile/Endings

10. De la même manière, Gilles Dowek montre que l'introduction d'instruments informatiques de calcul a transformé les mathématiques en produisant une nouvelle forme de jugement analytique a posteriori: «[L]es calculs effectués avec des ordinateurs permettent d'établir des jugements analytiques a posteriori: analytiques car ils permettent de résoudre des problèmes mathématiques, et a posteriori car ils reposent sur une interaction avec la nature » (Dowek, 2011, p. 188). Or, si un jugement est a posteriori, c'est que sa vérité ne peut être décidée à l'avance, sans en avoir fait l'expérience.

11. Certains jeux vidéo semblent ne jamais révéler l'intégralité de leurs règles et la recherche de glitches, c'est-à-dire d'effets du code non prévus par les développeuses, que les joueuses peuvent utiliser à leur avantage, peut continuer pendant plusieurs décennies. De tels glitches continuent d'être découverts tous les jours et c'est ainsi que The Legend of Zelda, Ocarina of Time (Nintendo, 
1998) - un jeu sorti il y a vingt ans et destiné à durer une trentaine d'heures - a pu être fini en moins de dix-sept minutes en mars 2018 (source : https://www.speedrun.com/oot).

12. C'est parce que les règles des jeux vidéo ne sont pas publiques et manifestes qu'il est si difficile d'appliquer la théorie (mathématique) des jeux aux jeux vidéo. En effet, en théorie des jeux, on distingue les jeux à information complète (comme les échecs) - où l'on connaît toutes ses possibilités d'actions et celles de son adversaire - et à information incomplète (comme le poker) où l'on ignore une partie de ces informations. La plupart des jeux vidéo entrent dans une autre catégorie puisque l'on ne connaît même pas l'ensemble des règles qui vont décider de nos possibilités d'action du jeu.

13. Il ne s'agit ici que de renverser et de prolonger une comparaison traditionnelle que l'on trouve chez Hobbes qui considérait déjà qu' « il en est des lois [politiques] comme des lois du jeu » parce qu'elles doivent être acceptées par tous ceux qui vivent en société (Hobbes, 2000 [1651], p 510).

14. Le problème de cette définition étant que les joueurs ne ressentent pas cet apprentissage comme tel, mais plutôt comme un plaisir lié à l'immédiateté de la réaction de ce qui se passe à l'écran : j'appuie sur un bouton et immédiatement je vois ce que cela a comme effet.

15. La physique naïve (au sens de "naturelle ») désigne un ensemble d'intuitions que possèderait le sens commun, c'est-à-dire toute appréhension infra-théorique de la réalité physique, telles que «les objets ont une permanence physique », «un objet jeté en l'air ralentit puis retombe en accélérant ", « un objet solide ne peut être traversé par un autre objet solide», etc. Pour un aperçu global des différentes recherches qui entrent dans le champ de la physique naïve, voir Smith and Casati (1994).

16. Voir notamment Spelke (2007).

17. En réalité, même la question de savoir si ces dispositions sont innées fait débat. Concernant les données et les controverses sur ces capacités, voir Gopnik et Wellman (2012).

18. Sur la manière dont des jeux vidéo comme le jeu de conquête spatiale Kerbal Space Program (Squad, 2011) ont pu être utilisés dans les salles de classe, voir (Garcin, 2014).

\section{RÉSUMÉS}

L'objectif de cet article est de reprendre les questions définitionnelles liées au jeu vidéo en montrant que si l'on s'est beaucoup interrogé sur le contenu de la définition du jeu vidéo, on s'est beaucoup moins préoccupé de sa forme. Je soutiens qu'une partie des difficultés qu'ont rencontrées les entreprises de définition du jeu et du jeu vidéo provient du type de définition adopté par la majorité des chercheuses ayant travaillé sur le sujet (les définitions explicites). En m'appuyant sur les résultats de la théorie de la définition, je propose de définir le jeu vidéo comme concept à texture ouverte et j'explore les différentes pistes de recherche qu'elle ouvre.

The goal of this paper is to examine problems related to the definition of video games. If previous analyses of this issue have focused on the content of this definition, few have investigated the form that such a definition should endorse. I argue that some of the problems encountered by these previous analyses come from the sort of definition most used in this context (explicit definitions). Drawing on the theory of definition, I suggest to define video game as an opentexture concept and I investigate several areas for further research based on this definition. 
INDEX

Mots-clés : jeu vidéo, règles, lois, définition, théorie de la définition, concept à texture ouverte

Keywords : video game, rules, laws, definition, theory of definition, open-texture concept

\section{AUTEUR}

\section{GAUVAIN LECONTE}

Enseignant au lycée de l'Europe, Dunkerque 